\title{
Visible Light and mmWave Propagation Channel Comparison for Vehicular Communications
}

\author{
Ali Uyrus, Bugra Turan, Ertugrul Basar and Sinem Coleri \\ Department of Electrical and Electronics Engineering, Koc University, Sariyer, Istanbul, Turkey, 34450 \\ E-mail: [auyrus18, bturan14, ebasar, scoleri] @ku.edu.tr
}

\begin{abstract}
Future connected vehicles are expected to require fast and reliable exchange of road information to increase safety and enable cooperative driving. Currently, standardized vehicular communication technologies aim to enable basic safety message exchanges with limited bandwidth. Recently, alternative technologies, based on millimeter-wave (mmWave) and visible light spectrum are proposed as complementary vehicle-toeverything (V2X) communication schemes, provisioned to support future connected vehicles with high bandwidth and increased security. However, the understanding of channel propagation characteristics is the key to achieve reliability, due to higher path loss compared to $5.8 \mathrm{GHz}$ band. In this work, we compare channel path loss characteristics of mmWave and vehicular visible light communication (VVLC) schemes to provide an overview regarding technology selection in an indoor parking garage. Path loss measurements are conducted with respect to various intervehicular distances, receiver angles, nearby vehicle existence, and lane occupation scenarios. Measurement results indicated path loss of $21.47 \mathrm{~dB}$ for VVLC from $3 \mathrm{~m}$ to $20 \mathrm{~m}$ distances. Moreover, path loss for $\mathrm{mmWave} 26.5 \mathrm{GHz}$ and $38.5 \mathrm{GHz}$ channels increased $12.5 \mathrm{~dB}$, and $12.7 \mathrm{~dB}$, respectively. Nearby vehicles are shown to decrease path loss of $26.5 \mathrm{GHz}$ and $38.5 \mathrm{GHz}$ signals up to $9.78 \mathrm{~dB}$, and $9.56 \mathrm{~dB}$, respectively, whereas VVLC channel path loss decreases $0.4 \mathrm{~dB}$ at the same scenario. Channel frequency response (CFR) measurements indicated frequency flat behavior of VVLC channels while mmWave channel exhibits frequency selectivity induced dispersion due to parking garage structure. Obstructed line-of-sight $(\mathrm{OLOS})$ measurements further reveal that blocking vehicle interrupts VVLC signals while selecting a favorable antenna location leads up to $30 \mathrm{~dB}$ less path loss for mmWave signals.
\end{abstract}

Index Terms - Vehicular communication, visible light communication, vehicle-to-vehicle communication, mmWave Vehicular Communication, vehicle-to-infrastructure communication

\section{INTRODUCTION}

Next generation connected and autonomous vehicles $(\mathrm{CAVs})$ are expected to be equipped with vehicle-to-vehicle (V2V) and vehicle-to-everything (V2X) communication systems providing fast and reliable data exchange with nearby vehicles and infrastructure to increase road safety. Current V2X communication technologies aim to support exchange of basic safety messages including vehicle size, location, heading, acceleration, speed, and weather data with limited bandwidth and latency. Therefore, applications requiring high bandwidth and low latency, such as raw sensor data sharing and see through, are not readily supported with standardized IEEE 802.11p and Cellular Vehicular to Everything Communication $(\mathrm{C}-\mathrm{V} 2 \mathrm{X}) \mathrm{V} 2 \mathrm{~V}$ communication technologies.
Recently, complementary V2X communication technologies, utilizing millimeter-wave (mmWave) and visible light communication (VLC) have been proposed to fulfill the requirements of next generation CAVs. When compared to current IEEE $802.11 \mathrm{p}$ and cellular based communications utilizing frequencies between $5.850-5.925 \mathrm{GHz}, \mathrm{mmWave}$ based schemes, enable utilization of higher bandwidths, whereas, VLC provides secure, line-of-sight (LoS), and radio frequency (RF) interference free communications. Therefore, both technologies can be considered as complementary V2V schemes providing the additional advantages of high bandwidth and secure communications for vehicular applications such as platooning [1], [2]. However, considering the high propagation path loss of both technologies, large scale fading characterization at practical $\mathrm{V} 2 \mathrm{X}$ communication usage scenarios is key for reliable system designs. Currently, channel models for V2X communications for mmWave frequencies, $30 \mathrm{GHz}$ and $63 \mathrm{GHz}$, targeting vehicle-to-base station (V2B), pedestrian-tobase station (P2B), and base station-to-road side unit (B2R) including urban and highway scenarios are detailed in [3], whereas, mmWave channel models for $\mathrm{V} 2 \mathrm{~V}$ and vehicle-topedestrian (V2P) communication applications are not specified. The mmWave vehicular communication schemes are explored to date for their characteristics at different frequencies [4]-[7]. In [4], the path loss characteristics of $60 \mathrm{GHz}$ radio waves are investigated on both metropolitan highways and regular roads; the authors of [5] studied V2V mmWave channel with time domain characteristics for antennas located near a vehicle's headlight. Ben-Dor et.al [6] explored 60 $\mathrm{GHz}$ outdoor channel characteristics considering in-vehicle antennas, whereas [7] evaluated $38 \mathrm{GHz}$ and $60 \mathrm{GHz} \mathrm{V} 2 \mathrm{~V}$ channel characteristics at a university campus. Moreover, [8] characterized the effects of vehicle blockage through measurements at $6.75 \mathrm{GHz}, 30 \mathrm{GHz}, 60 \mathrm{GHz}$, and $73 \mathrm{GHz}$ in both urban and highway scenarios. The authors in [9] characterized $28 \mathrm{GHz}$ channel with respect to vehicle blockage in an open parking lot.

Vehicular visible light communication (VVLC) channel characteristics are addressed only in a few studies. Specifically, [10] conducted VVLC Channel Frequency Response (CFR) measurement-based channel modeling, [11] proposed geometry-based stochastic model for VVLC, [12] investigated VVLC received signal power variations with respect to vehicle movements, and [13] provided an analytical expression to compute received signal strength (RSS) of VVLC channel. 


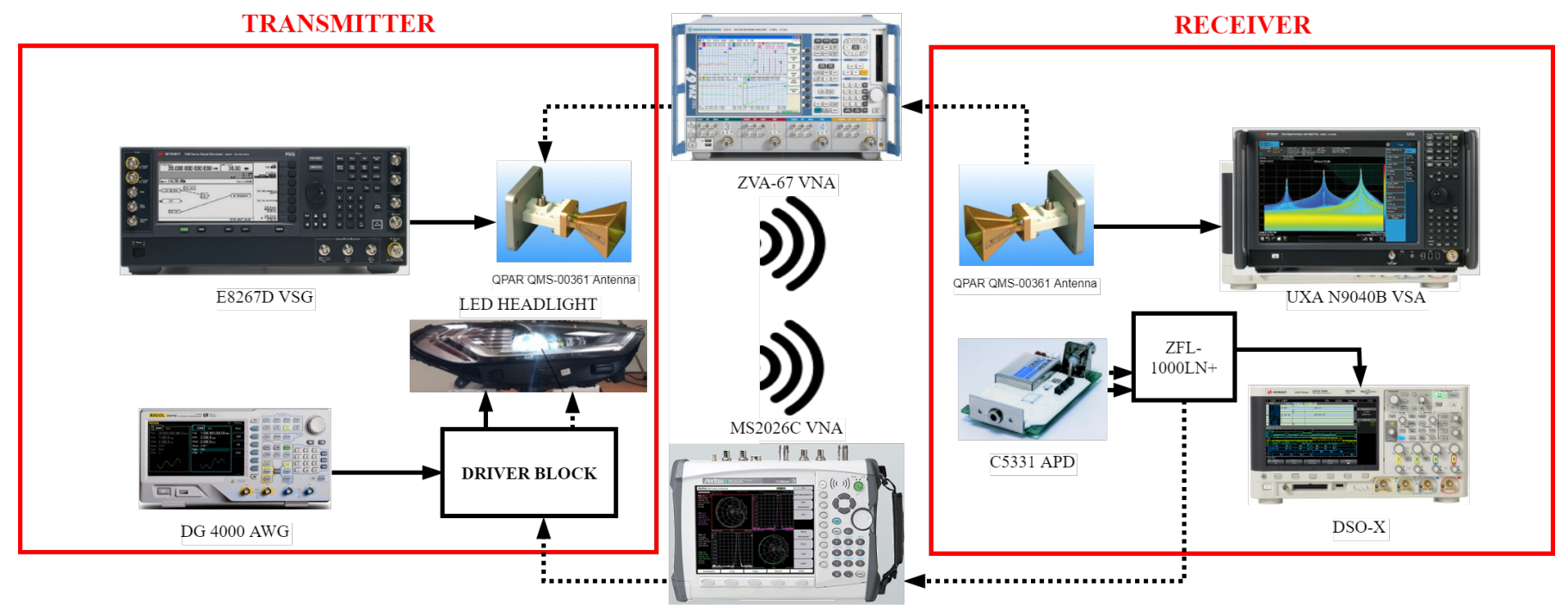

Figure 1: Experimental Setup

In this work, channel path loss comparisons between mmWave and VVLC bands targeting direct V2V or V2P communications in an indoor environment are performed. To the best of our knowledge, this is the first study in which mmWave and VVLC channel comparisons are performed to provide an overview on the vehicular communication technology selection in an environment comprising a parking garage. We utilize $26.5 \mathrm{GHz}$ and $38.5 \mathrm{GHz}$ frequencies for mmWave bands considering vehicle user equipment using 5G-New Radio Frequency Range 2, n257 and n260 bands [14]. Time domain channel path loss measurements are conducted in the -1 level of a 2 story underground parking garage with up to $28 \mathrm{~m}$ intervehicular distances, to investigate nearby vehicles, building columns, floor slabs, and wall effects on signal propagation. Moreover, CFR measurements of the technologies under consideration are conducted to further evaluate the received power variations with respect to the building structure and nearby vehicles. Finally, vehicle blockage effects for mmWave signals in the target underground parking garage are characterized through different cross sections of a production vehicle at 11 different antenna locations.

The rest of the paper is organized as follows. In Section II, we briefly describe the experimental setups utilized to measure mmWave channel path loss, VVLC RSS, mmWave and VVLC channel frequency responses. In Section III, we provide measurement results and compare mmWave and VVLC channel path loss characteristics including vehicle blockage loss for mmWave channel targeting indoor scenarios. Finally, we conclude the paper in Section IV.

\section{EXPERIMENTAL SETUP}

Experiments are conducted to understand the channel loss propagation differences between mmWave and VVLC channels in an indoor environment. Transmitter and receiver front ends of both technologies (i.e., Light Emitting Diode (LED)-avalanche photodiode (APD) for VVLC, antennas for
mmWave bands) are located at the same height of $70 \mathrm{~cm}$, to provide fair comparison. For direct LoS measurements, both antenna boresights and optical transmitter-receiver front-ends are aligned. Our experimental setup is depicted in Fig. 1 and detailed in following subsections.

\section{A. mmWave Channel Measurement Setup}

MmWave signals at $26.5 \mathrm{GHz}$ and $38.5 \mathrm{GHz}$ are generated by Keysight E8267D Vector Signal Generator for channel path loss characterization. Generated signals are transmitted and received through QPar QMS-00361 wideband horn antennas, with $22^{\circ}$ to $41^{\circ}$ half power beam width, $14.3 \mathrm{dBi}$ and $14.7 \mathrm{dBi}$ gain at 26.5 and $38.5 \mathrm{GHz}$, respectively. Received signals are fed into Keysight UXA N0940B Vector Signal Analyzer, where received signal power levels are averaged and recorded for analysis.

CFR measurements for mmWave channel are conducted with Rohde Schwarz ZVA-67 vector network analyzer (VNA), where antennas are connected to two ports of VNA with Pasternack PE361 RF cables.

\section{B. VVLC Channel Measurement Setup}

A single tone sinusoidal signal at $1 \mathrm{MHz}$ is generated through Rigol DG4000 Arbitrary Waveform Generator, and fed into LED driver block. LED driver block includes a cascaded set of Mini-Circuits ZHL-6A+ and ZFL-500LN amplifiers with Mini-Circuits ZFBT-4R2GW+ bias tee. Amplified and DC-Biased signals are fed into a 2017 Ford Mondeo multibeam LED headlight (see Fig.3) from the output of the driver block.

At the receiver side, optical signals were captured through Hamamatsu C5331-03 APD and 20 dB amplified with MiniCircuits ZFL-1000LN+ amplifier. Received signal amplitudes are measured with Keysight DSOX3034A Oscilloscope for further analysis. 


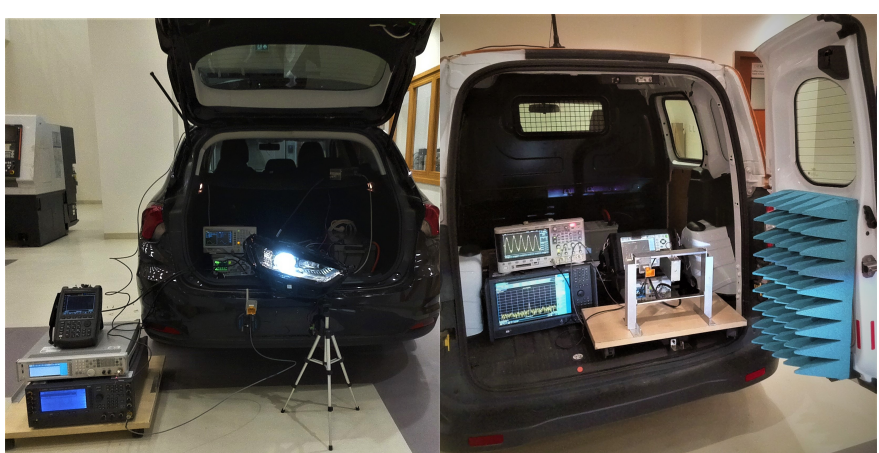

(a)

(b)

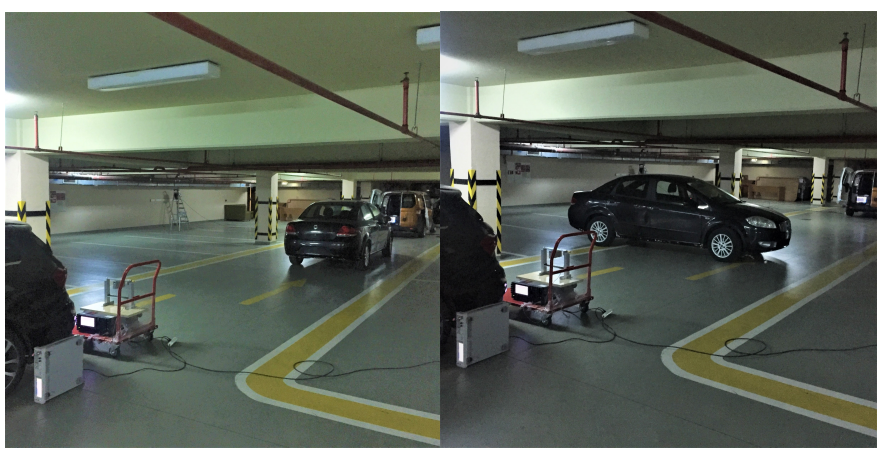

(c)

(d)

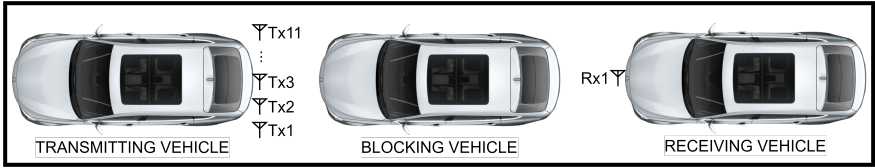

(e)

Figure 2: (a) Transmitter Setup, (b) Receiver Setup (c) and (d) obstructed line-of-sight (OLoS) scenarios for longitudinally and laterally blocking vehicles (e) OLoS Antenna Locations

Table I: VVLC Front End Specifications

\begin{tabular}{|c|c|}
\hline Parameter & Value \\
\hline \multicolumn{2}{|c|}{ Transmitter } \\
\hline Headlight 3-dB Bandwidth & $2 \mathrm{MHz}$ \\
\hline DC Bias Voltage & $24 V$ \\
\hline Driver Block Input Signal Amplitude & $63 \mathrm{~m} V_{p p}$ \\
\hline Driver Block Total Gain & $47 \mathrm{~dB}$ \\
\hline Driver Block Output Signal Amplitude & $14.1 V_{p p}$ \\
\hline LED Input Signal Amplitude & $5.6 V_{p p}$ \\
\hline LED Optical Transmitted Power & $-6.72 \mathrm{dBm}$ \\
\hline Transmitter Height & $0.7 \mathrm{~cm}$ \\
\hline \multicolumn{2}{|c|}{ Receiver } \\
\hline Avalanche Photodiode Module & Hammamatsu C5331-03 \\
\hline APD Active Area & $1 \mathrm{~mm}$ \\
\hline APD 3 dB Frequency Bandwidth & $4 \mathrm{kHz}$ to $100 \mathrm{MHz}$ \\
\hline APD Spectral Response Range & 400 to $1000 \mathrm{~nm}$ \\
\hline APD Peak Sensitivity Wavelength & $800 \mathrm{~nm}$ \\
\hline Amplifier & Mini-Circuits ZFL-1000LN+ \\
\hline Amplifier Gain & $20 \mathrm{~dB}$ \\
\hline Amplifier Frequency Range & 0.1 to $1000 \mathrm{MHz}$ \\
\hline Receiver Height & $0.7 \mathrm{~m}$ \\
\hline
\end{tabular}

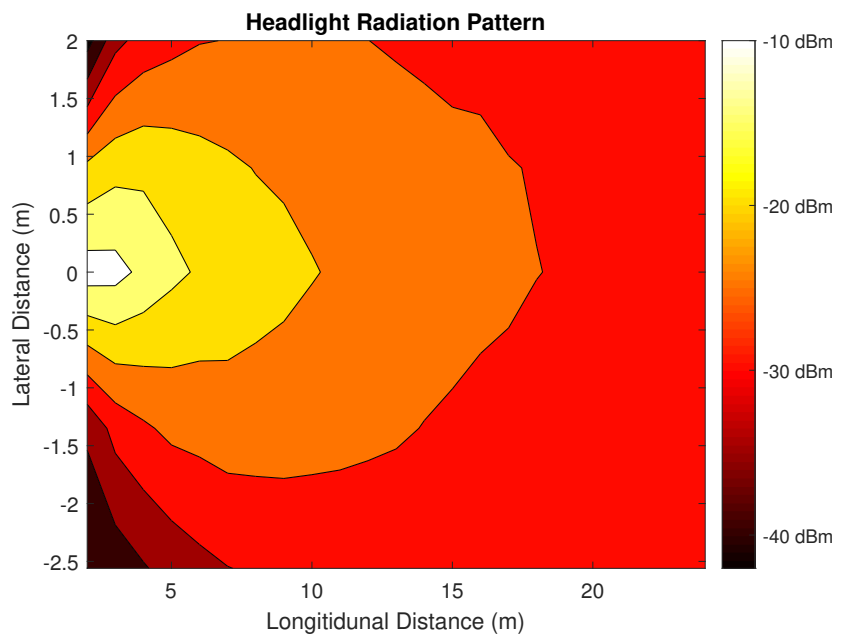

Figure 3: Headlight Radiation Pattern at $70 \mathrm{~cm}$ Height

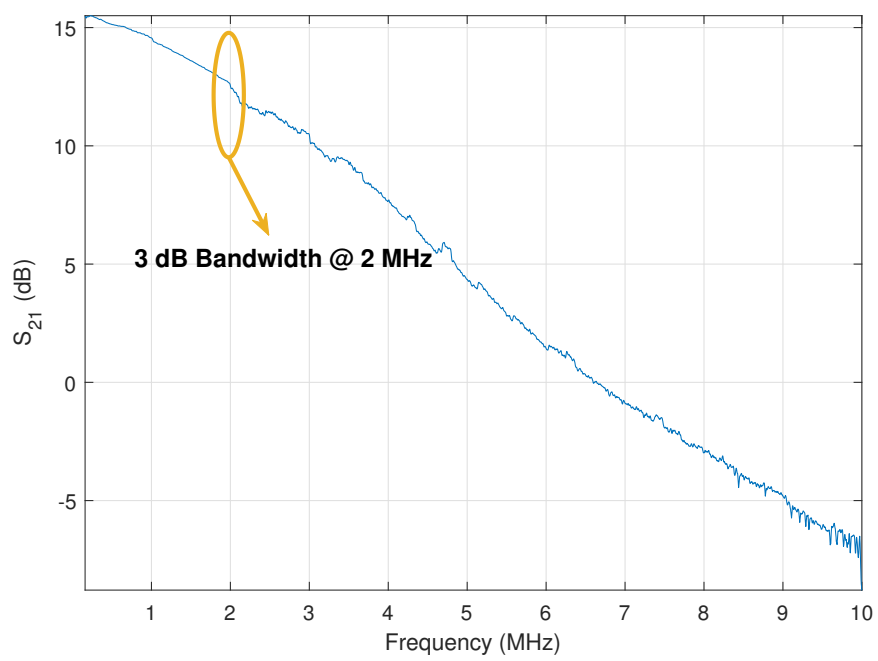

Figure 4: Headlight Frequency Response

Anritsu MS-2026C VNA is utilized for VVLC CFR measurements. LED Headlight is connected to the Port 1 of VNA through the driver block, where the amplified output of the APD through ZFL-1000LN+ amplifier is connected to port 2 of the VNA. VVLC CFR measurements are conducted between $200 \mathrm{kHz}$ and $10 \mathrm{MHz}$ due to limited modulation bandwidth of vehicle headlight power LEDs (see Fig.4). Table I depicts VVLC measurement setup specifications.

Table II: CFR Measurement Parameters

\begin{tabular}{lll}
\hline Parameters & VVLC & mmWave \\
\hline Frequency Range & $200 \mathrm{kHz}-10 \mathrm{MHz}$ & $25 \mathrm{GHz}-40 \mathrm{GHz}$ \\
Number of Points & 4001 & 4001 \\
Bandwidth & $100 \mathrm{kHz}$ & $100 \mathrm{kHz}$ \\
Cable Length & $7 \mathrm{~m}$ & $7 \mathrm{~m}$ \\
Tx Power & $-25 \mathrm{dBm}$ & $8 \mathrm{dBm}$ \\
\hline
\end{tabular}




\section{Measurement Scenarios}

1) Path Loss Measurements: For path loss measurements, one vehicle is equipped as mmWave and VVLC transmitter (Fiat Tipo, See Fig. 2(a)), while the other vehicle, Ford Courier, depicted in Fig. 2(b), is utilized as mmWave and VVLC receiver vehicle. Measurement scenarios are further detailed as follows.

a) One Lane Scenario: Vehicles are moved back-to-back at single lane in the parking garage to distinguish building structure effects such as columns (see Fig. 2(c) and Fig. 2(d)). Direct $\operatorname{LoS}$ and $30^{\circ}$ tilted receiver cases are considered for empty parking garage and full parking garage with nearby vehicles. Considering common $30^{\circ}$ tilt angle of production vehicle rear view cameras, optical receiver is oriented at $30^{\circ}$ angle to observe the inclination angle effects on VVLC link. On the other hand, nearby vehicle scenario (NBV) is explored to investigate tunneling effect of nearby vehicles in an indoor environment.

b) Two lane Scenario: Two lane scenarios are considered to compare both distance and angle dependent path loss of mmWave and VVLC channels for vehicles moving in opposite traffic directions.Two lane scenarios emulate weak received signal regions, as mmWave receiver antennas were out of boresight and VVLC transmitter LED emits light with less intensity due to its radiation pattern.

c) Blockage Scenario: Vehicle blockage induced path loss, OLoS, is measured for mmWave channel, where a third vehicle (Fiat Linea) is located between transmitter and receiver vehicles apart from $15 \mathrm{~m}$. For OLoS scenarios, 2 different cases, a blocking vehicle moving in the same direction with transmitter - receiver vehicles, longitudinal OLoS (Fig. 2(c)) and a vehicle located between transmitter - receiver vehicles, emulating a vehicle manoeuvring to leave from parking spot, lateral OLoS (Fig. 2(d)) are considered. Transmitter antenna is located on the rear bumper in 11 different locations at same height as shown in Fig. 2(e). All antenna locations are selected to be in the main beam of the receiver antenna, with negligible antenna gain differences. Thereby, mmWave channel path loss dependency on antenna locations are explored.

2) CFR Measurements: VVLC and mmWave CFR measurements are conducted to evaluate channel frequency characteristics with respect to varying locations covering different regions of the parking garage with pure walls and building columns. CFR measurements of mmWave and VVLC are executed simultaneously, where mmWave and VVLC transmitter - receiver front ends are located on two rolling platforms, as shown in Fig. 2(a). Both rolling platforms are moved in the parking structure, encapsulating one lane direct LoS path loss scenarios. Measured CFR $\left(S_{21}\right)$ parameters are recorded at static inter-vehicular distances from $1 \mathrm{~m}$ to $7 \mathrm{~m}$ with $1 \mathrm{~m}$ intervals for each measurement run due to cable limitations. Four measurement runs are executed for a total distance of $28 \mathrm{~m}$, covering all measurement points from direct LoS scenarios of path loss measurements. CFR measurement parameters are listed in Table II.
Table III: mmWave Channel Path Loss Parameters

\begin{tabular}{llllll}
\hline Frequency & Angle & NBV & $n$ & $\mu$ & $\sigma_{s}$ \\
\hline \multirow{2}{*}{$26.5 \mathrm{GHz}$} & \multirow{2}{*}{0} & No & 1.49 & 0.156 & 3.76 \\
& \multirow{2}{*}{30} & Yes & 1.31 & -0.22 & 3.48 \\
& & No & 1.51 & 0.179 & 4.611 \\
& \multirow{2}{*}{0} & Yes & 1.477 & 0.040 & 2.20 \\
\hline \multirow{3}{*}{$38.5 \mathrm{GHz}$} & & Yes & 1.581 & 0.075 & 4.23 \\
& \multirow{2}{*}{30} & No & 1.86 & -0.35 & 2.18 \\
& & Yes & 1.78 & -0.16 & 3.76 \\
& & & & & \\
\hline
\end{tabular}

\section{RESUlts AND Discussion}

\section{A. Path Loss and Received Power}

For $26.5 \mathrm{GHz}$ and $38.5 \mathrm{GHz}$ mmWave measurements, path loss is extracted, whereas received electrical signal power is recorded for VVLC, as exact transmit power, APD gains, optical-to-electrical and electrical-to-optical conversion efficiencies are not readily known. Path loss related parameters for mmWave channel, $P_{L}(d)$, are extracted from,

$$
P_{L}(d)=P_{L}\left(d_{0}\right)+10 n \log _{10}\left(\frac{d}{d_{0}}\right)+X_{\sigma}
$$

where, $P_{L}\left(d_{0}\right)$ is the path loss at the reference distance $d_{0}$ of $1 \mathrm{~m}, n$ is the path loss exponent, $d$ is the inter-vehicular distance at each measurement point, and $X_{\sigma}$ is a zero mean random variable which has Gaussian distribution with standard deviation $\sigma_{s}$. Linear regression with minimized mean squared error (MMSE) is utilized in order to obtain $n$ and $\sigma_{s}$ [15] for mmWave channel, and parameters are listed in Table III. Both $n$ and $\sigma_{s}$ values are observed to be consistent with the reported indoor mmWave channel path loss studies [16], [17], indicating wave guiding effects of indoor environment. Fig. 5 depicts that nearby vehicles, decrease path loss, while increasing tunneling effect. Furthermore, path loss is observed to decrease for one lane $30^{\circ}$ tilted receiver antenna scenario, likely due to reflections from parking garage floor slabs.

Path loss for mmWave channel and RSS for VVLC channels with respect to nearby vehicle and receiver angle variations for $5 \mathrm{~m}$ and $20 \mathrm{~m}$ are summarized in Table IV. Nearby vehicles together with $30^{\circ}$ receiver angle are demonstrated to decrease path loss up to $10.69 \mathrm{~dB}$ for mmWave channels, clearly indicating waveguide effect sourced from building floor and vehicles. VVLC RSS is observed to consistently decrease with tilted receiver, and increase with nearby vehicles.

Fig. 6 shows the cumulative density function (CDF) of mmWave channel path loss, ranged from $-118 \mathrm{~dB}$ to -70.7 $\mathrm{dB}$ fitting to Gaussian distribution with $-96.5 \mathrm{~dB}$ mean and $8.66 \mathrm{~dB}$ standard deviation.

Fig. 7 depicts the VVLC RSS for one lane scenarios, where the mean received power decreases by $2 \mathrm{~dB}$ with $30^{\circ}$ tilted receiver, and nearby vehicles are observed to have very subtle incremental effect $(0.4 \mathrm{~dB})$ on VVLC RSS. Inter-vehicular distance $\left(d_{i v}\right)$, dependent path loss equations are derived for all scenarios, where relevant coefficients $\left(a_{1}, a_{2}, a_{3}, a_{4}\right)$ are listed in Table V.

$$
H_{0}\left(d_{i v}\right)=a_{1} e^{a_{2} d_{i v}}+a_{3} e^{a_{4} d_{i v}}
$$




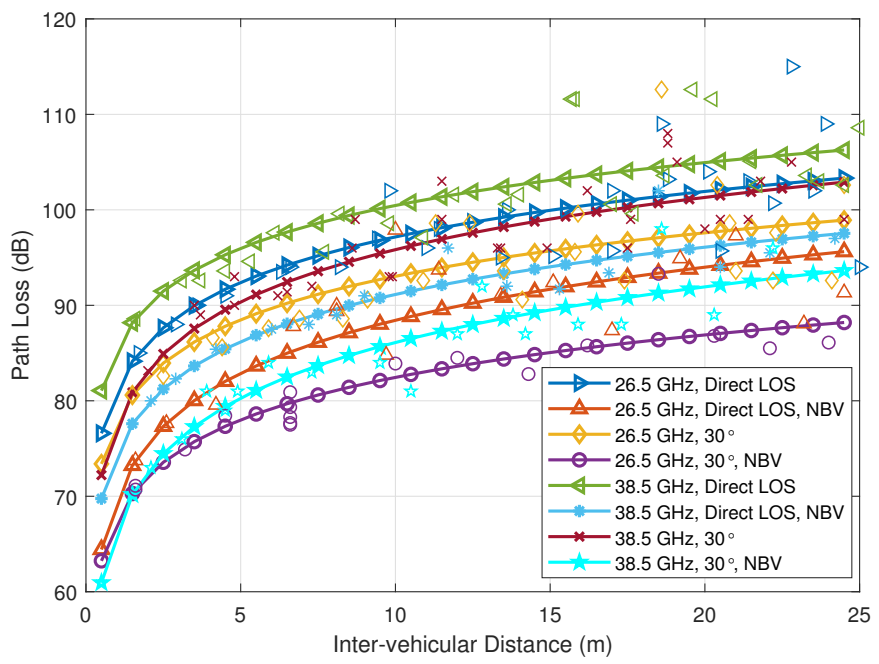

Figure 5: mmWave Channel Path Loss for 1-lane Scenario

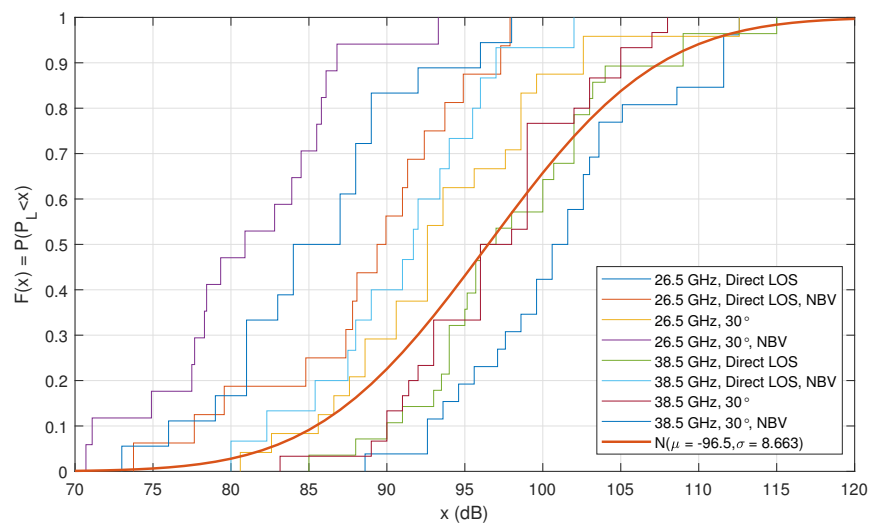

Figure 6: mmWave Channel Path Loss Empirical Cumulative Distribution Function Compared to Normal Distribution $(N(\mu, \sigma))$

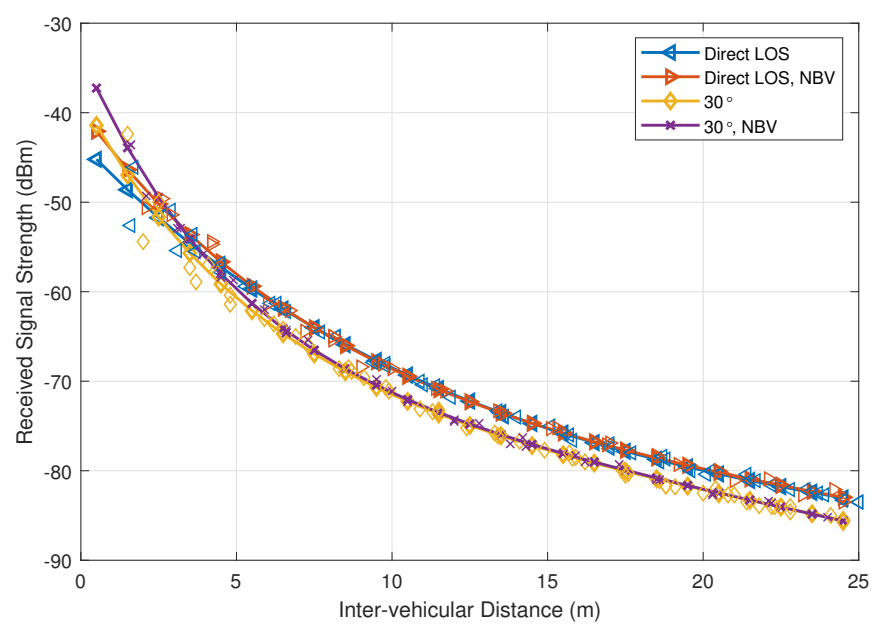

Figure 7: VLC Received Signal Strength for 1-lane Scenario
Table IV: Fitted Path Loss Comparison at $5 \mathrm{~m}$ and $20 \mathrm{~m} \mathrm{(dB)}$

\begin{tabular}{ccccc}
\hline Frequency & Distance & NBV & $0^{\circ}$ & $30^{\circ}$ \\
\hline \multirow{2}{*}{$26.5 \mathrm{GHz}$} & \multirow{2}{*}{$5 \mathrm{~m}$} & No & 92.41 & 88.50 \\
& \multirow{2}{*}{$20 \mathrm{~m}$} & Yes & 82.88 & 78.01 \\
& & Yo & 101.9 & 97.59 \\
& \multirow{2}{*}{$5 \mathrm{~m}$} & No & 95.97 & 86.90 \\
\hline \multirow{3}{*}{$38.5 \mathrm{GHz}$} & & Yes & 86.19 & 80.37 \\
& \multirow{2}{*}{$20 \mathrm{~m}$} & No & 104.9 & 101.3 \\
& & Yes & 96.09 & 91.93 \\
\hline \multirow{3}{*}{ VVLC (RSS (dBm)) } & \multirow{2}{*}{$5 \mathrm{~m}$} & No & -58.50 & -60.89 \\
& \multirow{2}{*}{$20 \mathrm{~m}$} & Yes & -58.06 & -59.72 \\
& & No & -79.97 & -82.12 \\
& & & -79.79 & -82.06 \\
\hline
\end{tabular}

Table V: VVLC Path Loss Coefficients

\begin{tabular}{lcccc}
\hline Scenario & $a_{1}$ & $a_{2}$ & $a_{3}$ & $a_{4}$ \\
\hline Direct LoS & -82.73 & 0.0023 & 39.33 & -0.0892 \\
Direct LoS w/NBV & -73.62 & 0.0056 & 33.9 & -0.1307 \\
$30^{\circ}$ & -71.13 & 0.0077 & 32.84 & -0.1817 \\
$30^{\circ}$ w/NBV & -69.92 & 0.0084 & 36.5 & -0.2036 \\
\hline 2-lane Direct LOS, $d>10 m$ & -27.97 & 0.0097 & -0.0021 & 0.3130 \\
2-lane 30,$d>10 m$ & -28.11 & 0.0126 & -0.00052 & 0.3430 \\
\hline
\end{tabular}

Measurement results from opposite traffic two lane scenarios are demonstrated for mmWave bands in Fig. 8(a) and VVLC in Fig. 8(b). VVLC two lane RSS measurements indicate that headlight radiation pattern has substantial effect on VVLC angular dependency, as same received signal power is obtained at $1 \mathrm{~m}$ and $25 \mathrm{~m}$ distances. On the other hand, 26.5 $\mathrm{GHz}$ and $38.5 \mathrm{GHz}$ path loss measurement results demonstrate that, at the out of boresight gain of antennas, nearby and surface reflections become dominant, as path loss decreases with $30^{\circ}$ tilted receivers when compared to direct LoS receivers.

\section{B. Channel Frequency Response}

CFR measurement results for mmWave bands are depicted on Fig. 9, where parking structure columns and nearby vehicle effects at various inter-vehicular distances are clearly demonstrated. However, VVLC CFR measurements, denoted in Fig. 10, imply frequency flat characteristics of VVLC channel, mainly shaped through LED frequency response, and channel gain mainly depends on inter-vehicular distances. Nearby vehicles are shown to increase channel gain while no substantial effects of parking structure columns are observed

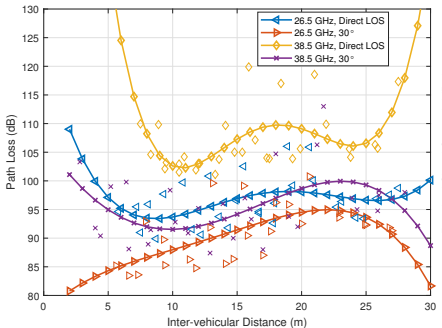

(a)

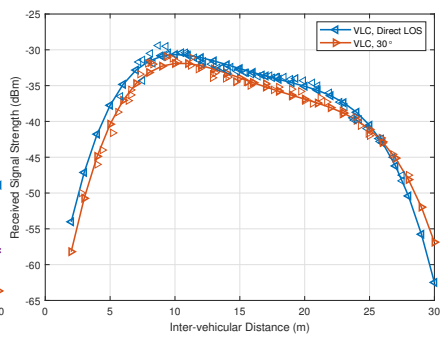

(b)
Figure 8: (a) mmWave path loss and (b) VLC received signal strength for 2-lane Scenario 

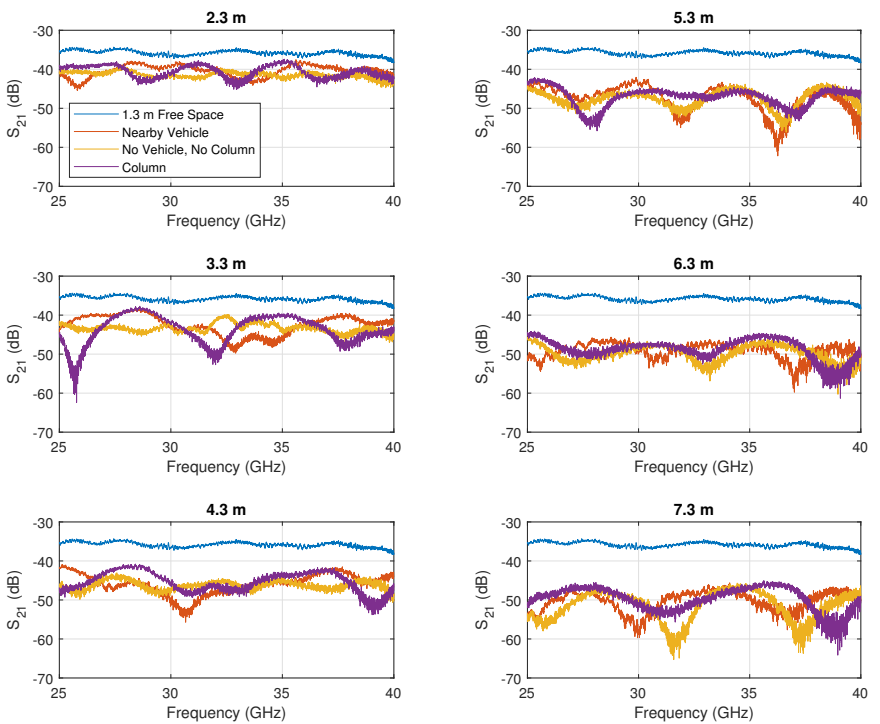

Figure 9: mmWave Channel Frequency Response
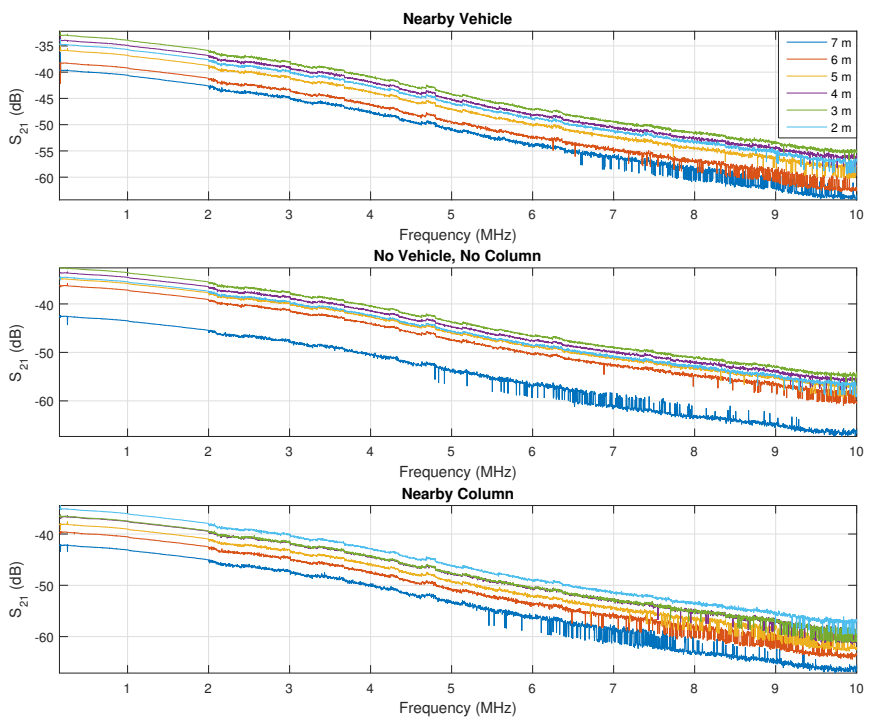

Figure 10: VVLC Channel Frequency Response

with VVLC CFR. Therefore, continuous channel estimation is important for mmWave channels, considering the building and nearby vehicle effects on the channel frequency domain response. However, as VVLC channels exhibit similar frequency response characteristics for various building structure and nearby vehicle existence, channel estimation periods can be extended, yielding less overhead, hence, lower latency.

\section{OLoS with Vehicle Blockage}

Vehicle blockage effects on $\mathrm{V} 2 \mathrm{~V}$ communication signal loss is investigated, where a third vehicle is located between transmitter and receiver vehicles. VVLC transmissions were not captured at the receiver with the blocking vehicle despite the high sensitivity of the receiver. Therefore, vehicle blockage can be regarded to interrupt VVLC transmissions.

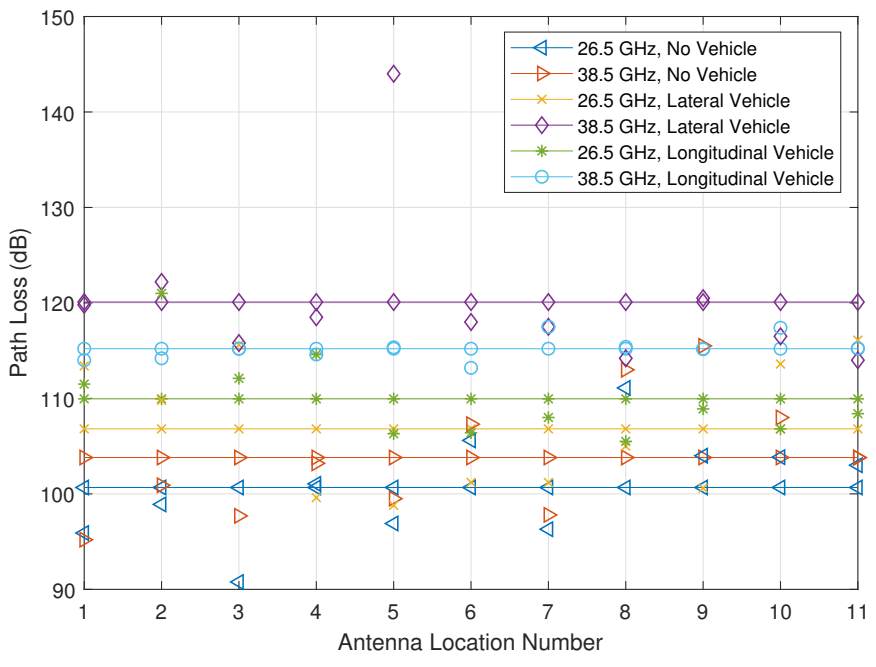

Figure 11: OLoS Path Loss

Table VI: OLoS Measurement Statistics

\begin{tabular}{llcc}
\hline Frequency & Scenario & $\mu$ & $\sigma$ \\
\hline \multirow{3}{*}{$26.5 \mathrm{GHz}$} & No Vehicle & 100.67 & 5.63 \\
& Lateral & 106.81 & 6.95 \\
& Longitidunal & 109.95 & 4.62 \\
\hline \multirow{3}{*}{$38.5 \mathrm{GHz}$} & No Vehicle & 103.81 & 6.53 \\
& Lateral & 120.09 & 8.33 \\
& Longitidunal & 115.21 & 1.30
\end{tabular}

Fig. 11 displays the path loss for one lane scenarios with a blocking vehicle, where the transmitter antenna location is varied at same height. Mean path loss $\mu$, for each OLoS scenario is depicted with solid lines in Fig. 11 and values are listed in Table VI along with standard deviations $\sigma$. Results indicate that path loss fluctuates up to $30 \mathrm{~dB}(38.5 \mathrm{GHz})$ with laterally located blocking vehicle, even though longitudinal vehicle blockage leads maximum $15.5 \mathrm{~dB}$ path loss fluctuation.

\section{CONCLUSION}

In this paper, we have empirically compared propagation characteristics of mmWave and VLC technologies for $\mathrm{V} 2 \mathrm{~V}$ communication scenarios in an indoor parking garage environment with empty and full cases. Our measurement results indicate that both mmWave and VVLC signals take advantage of full parking scenario case with decreased path loss, due to nearby vehicles. Moreover, $30^{\circ}$ tilted receiver has been demonstrated to decrease path loss for mmWave signals, while decreasing RSS for VVLC. CFR measurements have indicated that, VVLC channel exhibits frequency flat behavior, while mmWave CFR varies with respect to building columns. Considering $12.7 \mathrm{~dB}$ direct LoS path loss increase for mmWave channel at $38.5 \mathrm{GHz}$, and $21.47 \mathrm{~dB}$ path loss for VVLC from $3 \mathrm{~m}$ to $20 \mathrm{~m}$ inter-vehicular distances, with penetration capability through blocking vehicles, mmWave communications can be regarded more reliable for indoor parking garages, with symmetrical walls. On the other hand, VVLC can be utilized more reliably for more complex parking 
garages with additional building columns. Regarding LoS optical propagation characteristics of VVLC, and directional antenna usage for mmWave bands, two lane scenario measurements depicted that angular pattern of vehicle LED is key for the performance of VVLC, whereas mmWave signals take advantage of floor slab reflections at $30^{\circ}$ tilted receiver.

CFR measurement results imply that VVLC channel exhibits frequency flat characteristics, despite the building structure with columns and walls, whereas frequency selective characteristics of mmWave signals play an important role for path loss. OLoS measurement results indicate that VVLC signals are completely shadowed through blocking vehicles, whereas lateral and longitudinal blocking vehicles lead different path loss for mmWave V2V communications. Furthermore, different antenna locations at same height is demonstrated to have substantial effect on mmWave channel direct and OLoS channel path loss, which can be further considered for diversity schemes.

\section{ACKNOWLEDGEMENT}

The authors acknowledge the support of Ford Otosan, 5G Valley Open Test Site (5G VATS) and Spark Measurement Technologies. E.Basar acknowledges the support of Turkish Academy of Sciences GEBIP programme.

\section{REFERENCES}

[1] J. Choi, V. Va, N. Gonzalez-Prelcic, R. Daniels, C. R. Bhat, and R. W. Heath, "Millimeter-wave vehicular communication to support massive automotive sensing," IEEE Commun. Mag., vol. 54, no. 12, pp. 160167, 2016

[2] S. Ucar, S. C. Ergen, and O. Ozkasap, "IEEE 802.11 p and visible light hybrid communication based secure autonomous platoon," IEEE Trans. Veh. Tech., vol. 67, no. 9, pp. 8667-8681, 2018.

[3] "3GPP TR 37.885 v15.3.0 Study on evaluation methodology of new Vehicle-to-Everything V2X use cases for LTE and NR,' Jun. 2019.

[4] S. Takahashi, A. Kato, K. Sato, and M. Fujise, "Distance dependence of path loss for millimeter wave inter-vehicle communications," in Proc. IEEE Veh. Tech. Conf. (VTC-Fall) (IEEE Cat. No. 03CH37484), vol. 1, pp. 26-30, IEEE, 2003

[5] E. Kampert, P. A. Jennings, and M. D. Higgins, "Investigating the V2V millimeter-wave channel near a vehicular headlight in an engine bay," IEEE Commun. Lett., vol. 22, no. 7, pp. 1506-1509, 2018.

[6] E. Ben-Dor, T. S. Rappaport, Y. Qiao, and S. J. Lauffenburger, "Millimeter-wave $60 \mathrm{GHz}$ outdoor and vehicle AOA propagation measurements using a broadband channel sounder," in Proc. IEEE Glob. Telecommun. Conf. (GLOBECOM, pp. 1-6, 2011.

[7] M. G. Snchez, M. P. Tboas, and E. L. Cid, "Millimeter wave radio channel characterization for $5 \mathrm{G}$ vehicle-to-vehicle communications," Measurement, vol. 95, pp. $223--229,2017$.

[8] M. Boban, D. Dupleich, N. Iqbal, J. Luo, C. Schneider, R. Müller, Z. Yu, D. Steer, T. Jämsä, J. Li, et al., "Multi-band vehicle-to-vehicle channel characterization in the presence of vehicle blockage," IEEE Access, vol. 7, pp. 9724-9735, 2019.

[9] J.-J. Park, J. Lee, J. Liang, K.-W. Kim, K.-C. Lee, and M.-D. Kim, "Millimeter wave vehicular blockage characteristics based on $28 \mathrm{GHz}$ measurements," in Proc.IEEE Veh. Tech. Conf. (VTC-Fall), pp. 1-5, IEEE, 2017.

[10] B. Turan, G. Gurbilek, A. Uyrus, and S. C. Ergen, "Vehicular VLC frequency domain channel sounding and characterization," in Proc. IEEE Veh.Netw.Conf. (VNC), pp. 1-8, Dec. 2018.

[11] A. Al-Kinani, J. Sun, C.-X. Wang, W. Zhang, X. Ge, and H. Haas, "A 2-D non-stationary GBSM for vehicular visible light communication channels," IEEE Trans. Wireless Commun., vol. 17, no. 12, pp. 79817992, 2018.
[12] A. Chen, H. Wu, Y. Wei, and H. Tsai, "Time variation in vehicleto-vehicle visible light communication channels," in Proc. IEEE Veh.Netw.Conf. (VNC), pp. 1-8, Dec. 2016.

[13] A. Memedi, H.-M. Tsai, and F. Dressler, "Impact of realistic light radiation pattern on vehicular visible light communication," in GLOBECOM 2017-2017 IEEE Global Communications Conference, pp. 1-6, IEEE, 2017.

[14] "3GPP TS 38.101-2 User Equipment (UE) radio transmission and reception; Part 2: Range 2 Standalone Release 16," Jun. 2019.

[15] T. Rappaport, Wireless Communications: Principles and Practice. Upper Saddle River, NJ, USA: Prentice Hall PTR, 2nd ed., 2001.

[16] G. R. Maccartney, T. S. Rappaport, S. Sun, and S. Deng, "Indoor office wideband millimeter-wave propagation measurements and channel models at 28 and $73 \mathrm{GHz}$ for ultra-dense 5G wireless networks," IEEE Access, vol. 3, pp. 2388-2424, 2015.

[17] A. M. Al-Samman, T. Abd Rahman, and M. H. Azmi, "Indoor corridor wideband radio propagation measurements and channel models for $5 \mathrm{G}$ millimeter wave wireless communications at $19 \mathrm{GHz}, 28 \mathrm{GHz}$, and 38 GHz Bands," Wireless Commun. and Mobile Comput., vol. 2018, 2018.

[18] T. S. Rappaport, G. R. MacCartney, M. K. Samimi, and S. Sun, "Wideband millimeter-wave propagation measurements and channel models for future wireless communication system design," IEEE Trans. Commun., vol. 63, pp. 3029-3056, Sep. 2015. 